\title{
Interhotels der DDR: Einrichtung zwischen historischer Wahrheit und Dichtung Ein Forschungsbericht ${ }^{1}$
}

\section{Silvia Ulrich}

The following overview of GDR interhotels and their references to East German literature traces the secret and close relations between the FRG and the GDR during the decades of the Cold War. The thesis on the disappearance of hotel literature after the Second World War is critically examined and compared with literary evidence from the seventies to the nineties. The essay argues that interhotels represent the irreconcilable division between literature and power. Starting from the observation that the Central Committee of the SED was guided by Germany's luxurious grand hotel tradition, established in the Twenties, the following account concludes that if the interhotels have played a crucial role in overcoming the "Hallstein Doctrine", in contrast, the effort to "reach and overtake" has proven to be a real trap for the "workers' and peasants' state" in the course of three decades.

Keywords: Interhotels der DDR; Christa Wolf; Volker Braun; Rudi Strahl; Günter De Bruyn; Hotelliteratur; symbolisches Kapital.

\footnotetext{
${ }^{1}$ Ich möchte an dieser Stelle den KollegInnen Peggy Katelhön, Daniela Nelva und Gerhard Friedrich für die regen Gesprächen über dieses Thema danken, die mir tiefgründigen Recherchen von Forschungsmaterialien ermöglicht haben.
} 


\section{Kokettieren mit der Vergangenheit}

Das Grand-Hotel-Phänomen, das die deutsche Kultur in den Jahren der Jahrhundertwende bis hin in die späten zwanziger Jahre geprägt und eine "Hotel-Culture" geschaffen hatte (Vgl. Seger 2005, 2007; Matthias 2006), schien mit Ende des zweiten Weltkriegs zu Ende gegangen zu sein. Die These, eine Wiedergeburt der Hotelliteratur nach 1945 soll deshalb für unwahrscheinlich gehalten werden, weil sie kein soziologisches Phänomen mehr darstelle, so dass sie nur im Bereich der "Erinnerung" weiterleben kann (vgl. Seger 2005: 447-448), lässt sich jedoch von ihrer Ausnahme widersprechen: die der Interhotels der Deutschen Demokratischen Republik. Es handelt sich um die Luxus-Hotelkette der Regierung, bekannt als "Vereinigung Interhotel (VI) der DDR". Gegründet wurde sie in Berlin in den frühen 1960er Jahren auf Veranlassung des Zentralkomitees (ZK) der SED und bestand aus 4- bzw. 5-Sterne-Hotels.

Ihre Entstehung geht auf eine schwierige Zeit der DDRGeschichte zurück, da nämlich die 1949 gegründete Republik erst im Jahre 1972 - dank der Entspannungspolitik Willy Brandts -internationale Anerkennung als souveränen Staat fand (Bernhagen/Schlottke 1988: 66). Da Hotels zunächst in der früheren sowjetischen Besatzungszone (SBZ) dann in der DDR unter Volks- Genossenschafts- und Privateigentum standen - wobei Letzteres eher selten war - wurden sie von unterschiedlichen Trägern verwaltet. Dazu zählen die Handelsorganisation (HO), die Konsumgenossenschaften (KG) und eben die Vereinigung Interhotel - seit Mitte der 1970er Jahre in fünf Qualitätskategorien eingestuft. (vgl. Wolter 2009: 281) Die der VI zugehörigen Häuser waren die feinsten und exklusivsten. Einen Präzedenzfall hatte die VI 
sowohl in dem Feriendienst des Freien Deutschen Gewerkschaftsbundes (FDGB) als auch in der HO ebenso wie in der Einzelhandelskette Intershop $\mathrm{GmbH}$, was von der Kollaboration mit verschiedenen Ministerien ebenso wie Apparaten der DDR-Regierung zeugte:

Das Hotelwesen war in der DDR dem Ministerium für Handel und Versorgung, dort dem Bereich Binnenhandel und darin wiederum dem Konsumgüterhandel zugeordnet. Ginge es um Valutabeziehungen, hatte auch der Bereich Kommerzielle Koordinierung (KoKo) in Abstimmung mit dem Ministerium für Finanzen und dem Ministerium für Staatssicherheit Einflussmöglichkeiten. (Wolter 2009: 280)

Nach Kriegsende hatte die SBZ die extremen Folgen der Entnazifizierung Deutschlands erlebt, nämlich dadurch, dass sie eine unnachgiebige Einhaltung der Werte des Sozialismus mit einer von oben auferlegten Ideologie bezahlt und ihre Bürger in anspruchslose Lebensumstände gezwungen hat, während die Bundesrepublik, bald entlastet von Kriegsentschädigungen und unterstützt durch den Marshallplan, in der unmittelbaren Nachkriegszeit bereit war, dem Wirtschaftswunder entgegenzugehen, was zu einer schnellen Verbreitung des Reichtums führen sollte. Dies führte in Westdeutschland zur allmählichen Abschleifung von Klassenunterschieden, so dass früher exklusive Hotelreisen nun für einen großen Teil der BRD-Bürger ein erreichbares Ziel wurden. Der so entstandene Massentourismus in der BRD hat die Illusion geschaffen, dass jedem einzelnen Touristen Privilegien gewährt wurden, die einst einer kleinen Elite gehörten. Der Realsozialismus hingegen brandmarkte den Luxus als 
spätbürgerlichen Ausdruck kapitalistischer Prägung und verbannte ihn aus seinem Wertesystem. Sein Ziel war es nämlich, den Urlaub - und den Fremdenverkehr im Allgemeinen - zu einem Mittel $\mathrm{zu}$ machen, um das politische Bewusstsein der arbeitenden Kräfte zu prägen, indem Freizeit und Erholung als ein Recht jedes Werktätigen durch Angebot von Dienstleistungen begriffen wurden, die zu sehr niedrigen Kosten erbracht und damit für alle DDR-Bürger zugänglich sein sollten. Dies hatte freilich zur Folge, dass diese Dienstleistungen den Urlaub nicht im Zeichen der Distinktion entwickelten.

Der Urlaub sollte also zu einem wichtigen Pfeiler des Wiederaufbaus Deutschlands aus den Trümmern des Weltkriegs werden, obwohl auf ideologisch unterschiedlicher Basis, nicht nur in der BRD (vgl. Hoffmann 1961), sondern vor allem in Ostdeutschland, wo 1947 - zweiundeinhalb Jahre vor DDR-Gründung - der Feriendienst des FDGB entstand ${ }^{2}$.

Insbesondere der staatliche Tourismussektor als Zweig des Erholungswesens wurde zu einem wesentlichen Teil der Sozialversorgung, dessen Innovationscharakter in der Vergangenheit wurzelte: »Mit dieser Idee knüpfte der FDGB an Vorstellungen und Ziele der deutschen Arbeiterbewegung in der Weimarer Republik, den gewerkschaftlichen Tourismus in der UdSSR vor und nach 1945, aber auch - selbstverständlich ohne diesen Bezug zu

2 «Der Feriendienst bot seinen Urlaubern ein Paket aus Unterkunfts-, Verpflegungs- und Betreuungsleistungen an. [...] Die Mehrzahl der Reisen führte ins Inland. Auslandsreisen ergaben sich durch die geringen Kontingente an Reisen auf den Urlauberschiffen sowie durch Scheckaustausch mit den Gewerkschaftsorganisationen anderer sozialistischer Länder». (Wolter 2009: 190) Zu diesem ArbeiterFreizeitprogramm zählten bis zu den 1970er Jahren nicht Grand Hotels, sondern eher spartanische Ferienheime. 
erwähnen - an die "Kraft-durch-Freude"-Reisen des Nationalsozialismus an«. (Wolter 2009: 184f.) Der Staat kämpfte jedoch mit den ständig steigenden Kosten des staatlichen Erholungswesens, so dass sich ausländische Touristen - die sogenannten Devisenbringer - bald als wichtige Einnahmequellen erwiesen: Es wurde geschätzt, dass ein westlicher Tourist zehnmal mehr an die DDR bezahlt hat als ein östlicher Urlauber. (Heinrich 2016: 64)

In einem prinzipiell nicht konkurrenzfähigen Wirtschaftssystem wie der Planwirtschaft, das leider bald mit der wachsenden Auslandsverschuldung konfrontiert wurde, hatte die Notwendigkeit westliche Gäste mit einer starken Währung anzuziehen als Folge, dass die DDR auch ein innovatives, luxuriöses und exklusives Gastgewerbe entwickelte - und das in deutlichem Kontrast zu ihren Prinzipien. Interhotels, auch Valuta-Hotels genannt, wurden also bald zu Heterotopien ${ }^{3}$, d.h. »anderen Orten im eigenen Land ", einem Zwischenraum, wo öfters nur ausländische Währung (v.a. Valuta, d.h. D-Mark) umlief; sie galten als eine Art "Steueroase", die jedoch mehr mit der Ansammlung von Auslandskapital als mit Steuererleichterungen zu tun hatten (vgl. Delius/Lapp 1999: 48). Weder Gästen aus dem Westen - für die jene luxuriösen Häuser eher ein Aderlass waren statt eines Privilegs (vgl. Christ 1986: 2) - noch dem Volk des Arbeiterund Bauern-Staates standen sie zum echten Genuss da, sondern nur der DDR-Regierung zum (angeblichen) Profit. Interhotels erwiesen sich also von Anfang an als Blendwerk und Deckmantel zugleich.

So entstanden - neben dem berühmten Hotel Neptun in Warnemünde an der Ostsee - das übrigens niemals zur Interhotel-Kette, sondern zur HO gehörte (vgl. Pohlmann

${ }^{3}$ Vgl. Foucault 1967; Ulrich 2013, 2018. 
2017) - die ersten elf Strukturen aus den sechziger Jahren: eine in Berlin-Ost (Hotel Unter den Linden, seit 1966), in Erfurt (Erfurter Hof, seit 1965), in Jena (International, seit 1965) und in Magdeburg (International, seit 1965), zwei in Chemnitz (damals Karl-Marx-Stadt: Chemnitzer Hof, seit 1965; Hotel Moskau, seit 1962) und fünf in der Messestadt Leipzig (Astoria, seit 1949 als HO-Hotel, dann seit 1965 als Interhotel; Hotel Deutschland, seit 1964; Hotel Stadt Leipzig, seit 1964; International, seit 1949 als HO-Hotel, dann seit 1964 als Interhotel; Zum Löwen, seit 1965). Das Astoria in Dresden, umgebaut aus dem 1931 errichteten Haus der Jugend, eröffnete bereits 1950 und trat der VI erst 1965 bei. In Dresden übrigens befand sich auch das einzige von der VI betriebene erstklassige Motel (vgl. DDRLexikon).

Laut des Präfixes "Inter" spielt die Bezeichnung "Interhotel" auf eine internationale Öffnung an, die in Wirklichkeit nicht so oder zumindest nur unter besonderen Umständen so war. Denn sie wurde intern vom Ministerium für Handel und Versorgung verwaltet, während die ähnliche Präsenz im übrigen Ostblock nur das Ergebnis eines identischen Namens war. Die InterhotelKette ist daher eine Besonderheit der DDR und seine Internationalität besteht in dem Bedürfnis, Gäste aus Ländern jenseits des Eisernen Vorhangs (d.h. aus nichtsozialistischen Wirtschaftsgebieten) nach Ostdeutschland zu locken. Die ersten Strukturen lagen in der Nähe der Binnengrenze (deutsch-deutscher Grenze) und in Berlin-Ost, der Hauptstadt der DDR, mit dem doppelten Ziel, den Tourismus aus den westlichen Ländern und aus Russland abzufangen, um die jeweilige Währung abzuwerfen und die Gäste mit Spionageaktivitäten zu kontrollieren. Die Quellen sprechen auch von einer fiktiven Tauschwährung mit der D-Mark, die nur im Interhotel und 
in den dort vorhandenen Geschäften (den ebenfalls zur HO gehörenden Intershops) ausgegeben werden konnte, wodurch die Mitarbeiter und die einheimischen Bürger die in die luxuriösen Häuser aufgenommen wurden daran gehindert wurden, westliches Geld zu erwerben. Die Reaktionen des Volks darauf waren besonders hart, eben dort wo der internationale Charakter von Intershops und Interhotels ausposaunt wurde, die aber in der Tat nichts als die Unterlegenheit der DDR dem Westen gegenüber markierten. Bereits etliche Jahre vor der Entstehung von social networks gingen damals anonyme Meinungen in Zettelform über die Briefkästen mit kurzen, oft schlüpfrigen Spott-Gedichten. Daran erinnert sich Klaus Taubert in seinem Blog 4 wie folgend:

\section{Inter- Inter- Inter...}

Es stinkt so langsam schon zum Himmel, der so genannte "Inter-Fimmel",

als Intershop und Intertank, auch Interflug gibt's, Gott sei Dank.

In vielen Städten baut man heuer, Interhotels ganz groß und teuer.

Der große Knüller, den wir sah'n, steht an der Leipziger Autobahn.

Ein Parkplatz nur für West-Touristen, für den Fall, dass sie mal müssten!!!

$[\ldots]$

Im Laufe des Bestehens der DDR wuchs die Anzahl der Interhotels auf insgesamt 35. Nach der Wende wurden sie dann durch die Treuhandanstalt weitergeführt und 1992 an

4 https: / / klaustaubert.wordpress.com/2014/01/22/ddr-
spottgedichte/ 
die Klingbeil-Gruppe verkauft. Einige von ihnen existieren noch heute und gehören Hotelketten wie Mercure, NH, Hilton und Westin an; von ihrer Geschichte finden sich Spuren in der Presse ${ }^{5}$ und in wissenschaftlichen Büchern über Freizeit und Tourismus bzw. über politische und ökonomische Angelegenheiten ${ }^{6}$ in der DDR.

\section{Einholen ohne zu Überholen}

Der Luxus der Interhotels, der sonst von der Regierung ideologisch verpönt war, wurzelt in einem symbolischen Kapital historischer-kultureller Prägung. Die ersten Häuser gehen oft von bereits existierenden Etablissements aus, wie das 1915 in Leipzig gegründete Astoria, oder der 1905 in Erfurt eröffnete Erfurter Hof, dessen Ruhm sich dem Treffen zwischen BRD-Kanzler Willy Brandt und DDRMinisterpräsidenten Willy Stoph im Jahre 1970 verdankt. Weitere Beispiele sind das Hotel Zum Elefanten in Weimar und der Cecilienhof in Potsdam. Das erste wurde 1696 als Wirtshaus errichtet, 1938 nach Hitlers Plänen niedergerissen und erweitert wiederaufgebaut, schließlich als literarisches Hotel par excellence verewigt, mit einem zweifachen Sinn: Weil sich darin die Handlung von Thomas Manns Exilroman Lotte in Weimar (1939) abspielte und 1975 sogar deren Verfilmung durch Regisseur Egon Günter gedreht wurde; und weil Thomas Mann selbst als erster Gast nach dessen Wiedereröffnung als HO im Jahre 1950 im Elefanten abstieg. Der im Fachwerkstil 1917

\footnotetext{
${ }^{5}$ Vgl. Die Zeit (J. Lukas, Sieg der Sterne, 33/ 2016 vom 4.8.2016), Die Welt (B. Seipp, Was aus den Hotels des Sozialismus wurde, 26.10.2009, http: / / www.welt.de/4984328), der Spiegel (Wer wen melkt, 38/1990, S. 131-133)

${ }^{6} \mathrm{Vgl}$. Leitungsstile in der DDR
} 
errichtete Cecilienhof wurde hingegen 1960 als eins der ersten Devisen-Hotels eröffnet. Diese Beispiele zeigen, dass luxuriöse Hotels in der DDR mehrere Zwecke verfolgten: nicht nur verwiesen sie auf deren symbolisches und kulturelles Vermögen, sie sollten auch die DDR dem internationalen Ausland gegenüber symbolisch vertreten, vor allem in Bezug auf die nichtsozialistische Welt.

Dennoch blieben westliche Touristen in der DDR "Klassenfeinde" und galten daher als potenziell gefährlich für den Staat. Daraus ergab sich die Notwendigkeit, den westlichen Gast willkommen zu heißen und gleichzeitig auf ihn aufzupassen: In den Interhotels der DDR lebt die alte indoeuropäische Opposition auf, die die Konzepte von Gastfreundschaft und Feindseligkeit in demselben Gast vereint und überlagert, und sie schließlich zu den extremen Folgen führt (vgl. Benveniste 1976). Deshalb gelten Interhotels im historischen Gedächtnis weniger als Erholungs- denn als Spionageorte. Da diese Einrichtungen sich unter der direkten Kontrolle des Ministeriums für Staatssicherheit (MfS) befanden, stehen sie heute unter dem von der westlichen Presse geäußerten Verdacht, dass Gäste sogar in den Zimmern überwacht wurden. »Es ist aber unklar - meint Pohlmann (2017: 140), vorige Aussage relativierend - ob es überhaupt die technischen Voraussetzungen dafür gab, unbemerkt von den Gästen Videoaufnahmen in Hotelzimmern $\mathrm{zu}$ machen «. Um an Informationen zu gelangen, boten die Strukturen Gästen auch die Gesellschaft junger Frauen bzw. Männer mit guter Präsenz und Kultur an. Die sonst im gesamten deutschen sozialistischen Staat verbotene Prostitution wurde im Auftrag der Staatssicherheit in den Interhotels betrieben, geschützt und somit verwaltet (vgl. Spiegel 1976).

Unter diesem Blickwinkel erscheinen Interhotels als ein Beweis des Verrats des Staates an seinem Volk; eines 
janusköpfigen Staates mit Doppelmoral: im Äußeren sozialistisch, innerlich aber von echten marktwirtschaftlichen Prinzipien geprägt. Dies entging dem Volk nicht, das sich inzwischen über die schlimmsten Zustände des FDGB-Urlaubs beschwerte. (vgl. Wolter 2009: 192) Die Diskrepanz zwischen den Interhotels und den "strengen" Lebensbedingungen im Land (lange Reihen bzw. Wartezeiten, Versorgungsprobleme, engmaschige Kontrolle) führte schnellstmöglich $\mathrm{zu}$ wachsenden Protesten der DDR-Bürger, so dass das Politbüro des Zentralkomitees der SED Mitte der siebziger Jahre anordnete, dass sieben der Interhotels auch einheimischen Gästen, d.h. im Besitz von ostdeutscher Währung, die Türen öffnen. Zunächst war das erste Kontingent an Betten nur für »Angehörige volkswirtschaftlich wichtiger Betriebe« (Wolter 2009: 283) reserviert, die eine führende Rolle in der Wirtschaft des Landes spielten, ebenso wie für Arbeitnehmer, die sich durch besondere Verdienste ausgezeichnet hatten - Gewinnaufenthalte also, denen z.B. das Hotel Neptun in Warnemünde seinen Ruhm im Inland für viele Jahre verdankte. Ausgerechnet das Hotel Neptun zeugt vom Versuch seines Direktors Klaus Wenzel, den gleichzeitigen Aufenthalt von West- und Ostgästen in Einklang zu bringen (Christ 1986; Pohlmann 2017), z.B. durch Einführung einer Kunstwährung namens "Bewegungsgeld", die die Herkunft der jeweiligen Gäste nicht verraten sollte. 
Zeugnisse der Aufnahme von DDR-Gästen in den Interhotels enthalten widersprüchliche Bewertungen. Einerseits dienen sie der ideologischen Propaganda, wie folgende Schilderung eines Kurzurlaubs einer Familie im Interhotel Potsdam zeigt, vorgelegt vom jungen Sohn, und erschienen 1971 in der Hotelzeitschrift »Für Sie«:

... Papa ging uns anmelden und bekam den Zimmerschlüssel. ... und ich beschnupperte erst einmal das Hotel. Dann fuhren wir mit dem Lift in die 14. Etage. Katrin wollte gleich baden, Mami ausruhen und Papi Mittagessen. So hatte eben jeder seine Wünsche. Als es schon spät war, kamen wir wieder an unserem Interhotel an, und wir mussten ins Bett, nachdem wir vorher Abendbrot gegessen hatten. Ein Onkel, der Kollege Kellner hieß, brachte uns dauernd neue Teller, und Mutti brauchte nicht in die Küche rennen. Sie ging dann mit Papi in die Bar. Katrin und ich wollten auch in die Bar, aber Papa wollte mal ohne Jören sein. [...] Papa bezahlte das Wochenende. Er meinte, dass es gar nicht so teuer gewesen sei und dass man ruhig öfter verreisen solle ${ }^{7}$.

Der ausgewählte Kinderblick soll die Authentizität des Berichts als unbestreitbar glaubwürdig erscheinen lassen. Im Gegensatz dazu war es damals für die öffentliche Meinung ebenso unbegreiflich wie ärgerlich, dass die DDRRegierung einige Dienstleistungen (darunter Luxushotels und -läden) nur für ausländische Gäste, v.a. Westgäste, als

7 „Für Sie“ - Interhotels der DDR stellen sich vor, Herausgeber: Interwerbung $\mathrm{GmbH}$ Gesellschaft für Werbung und Auslandsmessen der DDR, Verlag Die Wirtschaft, 1971, zit. nach J. Fröhlich, Ein Hotel im Lustgarten von Potsdam. Das Interhotel Potsdam in historischer Umgebung von 1967 bis heute, BoD - Books on Demand GmbH, Norderstedt 2015 (3. verb. Aufl., 12012). 
Devisenbringer konzipierte, und dass westliche Investoren aus Schweden und Japan für deren Einrichtung beauftragt wurden (Potsdam, Dresden; Berlin; Warnemünde). In einem westlichen Zeitungsartikel aus dem Jahre 1986 (in dem die westliche Perspektive herrscht) wird berichtet, dass DDR-Bürger in Interhotel-Räumen eher toleriert als willkommen waren:

Wir waren im Dresdner Hotel "Bellevue" verabredet: zwei DDR-Freunde und ich. [...] Die Freunde spürten, $\mathrm{da} ß$ sie nicht gern gesehen waren und mißtrauisch und mißbilligend beäugt wurden. Als der Besucher aus Hamburg endlich auftauchte, atmeten sie auf. Sie wollten nichts wie weg, raus aus diesem Hotel, in dem sie nicht gelitten waren (Christ 1986: 1).

Da die »DDR-Freunde « die Pracht des Interhotels für dekadent hielten (»deren Preisliste sie gleich einsteckten als Dokument westlicher Dekadenz, gefördert vom Arbeiterund-Bauern-Staat«, ebd.), erschienen sie der Hotelführung offensichtlich als Spielverderber.

Noch verdächtiger wirkt die Vorstellung der InterhotelKette selbst, die man dem offiziellen Katalog entnehmen kann ${ }^{8}$ :

${ }^{8}$ Online-ressource: http:/ / www.ddr.center/katalogseite/interhotelsseite_1.html (letzter Aufgriff: 8.11.2019). 


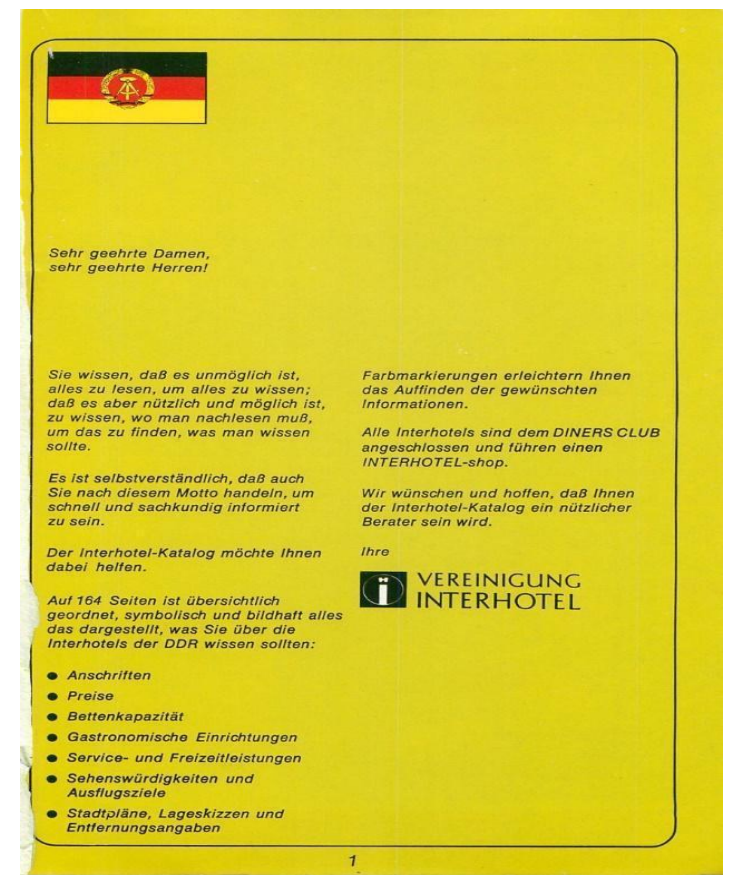

Hier verbirgt sich eine Warnung davor, in die Rolle des "kindlichen" Touristen $\mathrm{zu}$ fallen (»Sie wissen, dass es unmöglich ist, alles zu lesen um alles zu wissen «). Denn zwischen den Zeilen liest man, dass der Gast sich selbst und andere nicht $z u$ viel fragen sollte (»daß es aber nützlich und möglich ist, zu wissen, wo man nachlesen muß, um das zu finden, was man wissen sollte«), nämlich, dass Neugier nicht toleriert und womöglich bestraft wird (»Es ist selbstverständlich, dass auch Sie nach diesem Motto handeln«, Herv. [S.U.]).

Dies zeigt, dass Interhotels eine problematische Einrichtung selbst für die Regierung waren, weil sie eine Schwäche des sozialistischen Systems darstellten, v.a. was 
Spionage und Valuta betrifft - etwas, das der Historiker Matthias Judt in einem Interview von 2014 mit folgender Parole zusammenfasste: „Ökonomie siegt immer über Prinzipien ${ }^{9}$.

\section{"Die Hoffnung lag im Weg wie eine Falle"}

Abgesehen von vereinzelten Beispielen aus der trivialen Belletristik ${ }^{10}$, wird Interhotels in der Literatur keinen großen Raum gewährt. Warum? Waren Interhotels mit ihrem dekadenten Potential lauter Projektionen einer nach 1945 abgelehnten Vergangenheit und wurden somit als Last empfunden? Zweifellos sind sie ein "heikles" Thema, da sie vielmehr die Widersprüche der (Markt)Wirtschaft als die der Gesellschaft zum Inhalt haben. Dies könnte der Grund dafür sein, weshalb Schriftsteller beider damaligen

\footnotetext{
${ }^{9}$ A. Dunte, Matthias Judt im Interview: "Ökonomie siegt über Prinzipien". «Leipziger Volkszeitung» 1.03.2014, S. 3.

${ }^{10} \mathrm{Im}$ self publishing und über das Netz erhältlichen Roman Poppichs Flucht (22015) des Romanciers Herbert Größe spielt der erste Teil der Handlung im Interhotel in Magdeburg. Der Protagonist Paul Thiele, Kellner im Interhotel, zeigt die Welt der Mitarbeiter und deren Umgang mit dem MfS, das ihm schließlich zu betrügen gelingt, indem er sich selbst und 4 anderen Kollegen die Flucht bereitet und verhilft. Das Hotel als symbolische Flucht vor der engen Realität der DDR ("Sobald Paul das Interhotel betrat, verließ er die graue Alltagswelt der DDR und tauchte in eine andere Welt ein", Große 2015: 8) wird also zunächst zum Spiel, dann zum ernsthaften Unternehmen. Dort erscheint das Interhotel als eine DDR-Adaption von Thomas Manns Felix Krull, vor allem von dessen parodistischer Funktion ("Das Interhotel war wie ein Kokon. Außen und in der ersten Hälfte des Restaurants war es die typische DDR. Ab der zweiten Hälfte des Restaurants, in der Hotelhalle und in den Konferenzzimmern galten andere Regeln", Große 2015: 6), so zumindest verspricht der relativ niedrige Erwartungshorizont des Textes, in dem weitere Werke widerhallen, z.B. Delius Spaziergang von Rostock nach Syrakus (1995), oder Kafkas Amerika (1927).
} 
deutschen Staaten ihnen nur episodisch die richtige Aufmerksamkeit in ihren Werken schenkten. Intellektuelle der DDR haben sich durch ihr literarisches Schaffen ständig bemüht, »die Zukunft immer wieder zu erfinden « (vgl. Sisto 2009: 11), während die politische DDR-Führung (nämlich das ZK der SED) durch die Gründung der Vereinigung Interhotel lieber die Vergangenheit einverleibt hat. Als kapitalistische Zellen im Leib der Planwirtschaft, als Keime und tödliche Metastasen in einem angeblich gesunden Körper wiederholen Interhotels die unheilbare Trennung von Literatur und politische Macht. Von diesem Aspekt zeugen viele Werke der DDR-Literatur; einige von Ihnen bedienen sich des Interhotel-Bildes, um diese Trennung zu veranschaulichen.

So z.B. Christa Wolfs Roman Kindheitsmuster (1976: 589), deren Bemühungen, beide Sphären als eng miteinander verflochten darzustellen, durch das Interhotel-Episode erfolgreich zum Vorschein kommen. Anlässlich einer Reise nach der Heimatstadt Landsberg an der Warthe, unternimmt die Protagonistin (sprich: Wolf selbst) eine Reise in ihre eigene Vergangenheit, als sie nämlich eine vom Nationalsozialismus geprägte Erziehung erhielt. Dass sie ausgerechnet in einem Interhotel in Frankfurt an der Oder speist, zeugt von der Absicht, die Raum-Zeit- Ebenen "Hotel mit westlichem Flair" und "Vergangenheit" mit dem Faschismus in Verbindung zu bringen. Dort musste sie sich übrigens mit einer Reisegruppe aus Portugal konfrontieren, was ihre Kritik am kapitalistischen Ausland auslöste, das ihr als Ebenbild und Symptom von Faschismus auch in der Gegenwart erschien:

Im Interhotel von Frankfurt (Oder) bringt man euch ein vorzügliches Essen, wenn ihr darauf auch lange warten müßt, weil eine westliche Reisegesellschaft fast das 
ganze Lokal besetzt hat und alle Kellner in Anspruch nimmt. Ihr könnt die Sprache der teuer und papagaienhaft gekleideten, meist älteren Leute nicht ausmachen. Portugiesen, sagt die Kellnerin verächtlich. Geben an wie Graf Koks. - Die Männer haben schwere Augenlider und schlaffe Züge, die Frauen grell geschminkte, scharfe Gesichter. Viel Gold an Händen und Hals. Ob die sich selber schön finden, fragt sich Lenka. Ihr Onkel, Bruder Lutz, gibt ihr den guten Rat, die seltene Gelegenheit $\mathrm{zu}$ nutzen und sie lebende Exemplare einer herrschenden bürgerlichen Klasse anzugucken. - Das gibt mir nichts, sagte Lenka. - Heute, keine vier Jahre später, werden die Ergebnisse der ersten Wahlen nach der Niederschlagung des Faschismus in Portugal kommentiert. (Wolf 2000: 589-590)

Die Wahlen, auf die Wolf hier Bezug nimmt, hatten eine Steigerung der Rechtskonservative erlebt - ein Szenario, das sie assoziativ unmittelbar mit der Reisegesellschaft im Interhotel und mit den eigenen Erinnerungen an die NSVergangenheit in Verbindung setzt.

Trotz Mangels an erzählerischen Werken mit Interhotels als Schauplatz von fiktionalen Geschehnissen - im Unterschied zur Literatur der Jahrhundertwende und der zwanziger und dreißiger Jahre ${ }^{11}$ - gelten Interhotels als Orte fragwürdiger Werte und werden zum metaphorischen Pendant der Handlung. So z.B. in Volker

11 Berühmte Beispiele sind T. Manns Tod in Venedig (1911) und Bekenntnisse des Hochstaplers Felix Krull (1954), A. Schnitzlers Fräulein Else (1924), S. Zweigs Schachnovelle (1942) und Rausch der Verwandlung (posth. 1981), J. Roths Hotel Savoy (1924), V. Baums Menschen im Hotel (1929). Vgl. dazu die bereits erwähnten Studien von Seger 2005, 2007 und Matthias 2006, sowie Ulrich 2013, 2018 (in dt. Spr.) und 2010 (in it. Spr.) 
Brauns Unvollendete Geschichte (1977), in der sich eine zwar flüchtige doch mit Bedeutung aufgeladene Erwähnung lesen lässt. Die Protagonistin Karin, Tochter eines Parteifunktionärs, ist in den Staatsfeind Frank verliebt, was zur Bekämpfung von deren Liebesbeziehung durch ihre Familie führt. Nachdem Karins Vater seiner Tochter den Umgang mit Frank untersagt hat, ohne ihr den wahren Grund zu nennen - d.h. Franks Unfügsamkeit dem System gegenüber - versucht Karin vergeblich, sich von ihm zu trennen; stattdessen fühlen sich beide voneinander angezogen, und da sie ihre Geborgenheit nirgendwo genießen dürfen, wählen sie dafür ein Interhotel:

Sie machten sich verrückt, sie hielten nicht mehr aus. Sie gingen hinaus, Wind und Matsch, liefen den Platz hinüber zum Interhotel. Karin wollte nicht mit Frank auf dasselbe Zimmer eingetragen sein, sie verlangten zwei. Als die Frau an der Reception die Personalien prüfte, verweigerte sie Frank die Unterkunft. Er sei in der Stadt polizeilich gemeldet, könne also kein Hotel benutzen. Sie setzten sich ins Restaurant und aßen etwas. Dann ließ Karin Frank nach oben gehen in ihr Zimmer, mit ihrem Schlüssel, sie kam paar Minuten später nach. Sie legten sich ins Bett. Sie fingen wieder an, zu überlegen. Karin küßte ihn auf die Brust, auf den Leib, und er legte sich auf sie und drang in sie ein. Aber er dachte sogleich wieder nach und war nicht bei sich, und fühlte plötzlich, daß er nicht mehr in ihr war und sich nur so bewegte. Er erschrak, und es war erst mal vorbei. Karin war das Bett zu weich, sie warfen die Zudecke auf den Boden und legten sich hinunter. Das Zimmer war überheizt, auf der Straße unten dröhnten die Fernlaster. Sie versuchten es wieder, sie quälten sich. Karin schlief endlich ein. Frank 
wälzte sich bis zum Morgen herum. (Braun 1977, S. 1819)

Im Zitat erscheint das Interhotel als echtes Modell für westliche Dekadenz, weil es in Verbindung mit Bildern von verbotener und illegaler Liebe gebracht wird. In diesem Ort des Verbotenen, wo es den Figuren unheimlich wird - das Bett ist $\mathrm{zu}$ weich, im Zimmer ist es zu heiß, die ersehnte Sexualintimität wird mit Schuldgefühl erlebt, im Gegensatz zu den draußen dröhnenden Fernlaster als Symbol emsiger DDR-Produktivität - fühlen sich die Protagonisten entpersönlicht. In der literarischen Fiktion der Novelle veranschaulicht dieser Ort den Wendepunkt der Beziehung zwischen Karin und Frank, die von nun an tragische Züge annimmt.

Ein ähnliches Beispiel des Zusammenhangs zwischen Interhotels und Eros führt Rudi Strahls Theaterstück Flüsterparty an, in dessen Handlung das Interhotel als Quelle harter Währung die Fantasien einer Gruppe von Jugendlichen aus einer DDR-Provinzstadt lockt und sie ein Mädchen in die Bar des hiesigen Interhotels zum "Anschaffen" schicken. 1978 geschrieben, wurde es unmittelbar vor der Uraufführung am Ost-Berlins Maxim Gorkij Theater zensiert, weil die dort angedeutete Ausübung der Prostitution in den Interhotels den führenden Genossen der Theaterintendanz missfiel (vgl. Pragal 2019: 393).

Ein nobler Versuch hingegen, durch die Literatur auf die politische Macht Einfluss zu üben, wurde unternommen von $\gg 88$ Schriftsteller[n], Künstler[n] und Wissenschaftler[n]. 60 von ihnen stammen aus der DDR, sechs aus "weiteren sozialistischen Ländern, 22 aus der BRD, anderen nichtsozialistischen Ländern und BerlinWest"« (Berg 2014: 5), die sich in Berlin am 13.-14. 
Dezember 1981 im Interhotel Stadt Berlin am Alexanderplatz zu einem Friedens-Kolloquium trafen. Unter den Persönlichkeiten der deutsch-deutschen Literaturszene sind folgende zu erwähnen: Stefan Hermlin, der das Treffen organisierte, Jurek Becker, Thomas Brasch, Günter De Bruyn, Ingeborg Drewitz, Bernt Engelmann, Erich Fried, Günter Grass, Stefan Heym, Ernst Jandl, Heiner Müller, Christa Wolf. Diese »Begegnung zur Friedensförderung « (ebd.) gilt eher als Anlass der reziproken Verständigung denn als Inhalt fiktionaler Verarbeitung. Jedoch wird sie in ihrer als unwahrscheinlich empfundenen Wirklichkeit für ein »sozialistisches Märchen « (ebd.) gehalten, in dem unerhörte Gegebenheiten sich ereignen, wie die »Herstellung von Vertrauen « (ebd): »"Manchmal ließen sich", erklärt er [Hermlin, S.U.], „Dinge ganz einfach verwirklichen, die manche Leute für ausgeschlossen halten, weil sie nicht bereit sind, einen Versuch zu wagen“" «. (Berg 2014: 5) Auch in diesem Fall, wie bei Wolfs Kindheitsmuster, spiegelt sich das Geschehene im literarischen Bericht, von dem der damalige Jugendliche Stefan Berg - De Bruyns Verehrer Dreißig Jahre später als Erinnerung an jene Tage niederschreibt und veröffentlicht samt De Bruyns Briefwechsel mit ihm. Dieses Beispiel zeigt - wenn auch frei von jeglicher literarischer Fiktion - dass Schriftstellerei, Gesellschaft, Macht und Alltag untrennbar miteinander verflochten sind, und dass ein Interhotel die Verortung eines erweiterten literarischen Feldes darstellen kann.

Die heterotopische Funktion des Interhotels Stadt Berlin, an dem ostdeutsche und westdeutsche Schriftsteller sich einverständig mit Abrüstungsfragen auseinandersetzen und »die Blockkonfrontation abschwächen oder gar überwinden halfen « ist hier offensichtlich. (vgl. Berg 2014: 8) Der anfängliche Traum eines neutralen Deutschlands 
zwischen den Blöcken des kalten Krieges erlebt im deutschdeutschen Friedensdialog eine mögliche Realisierung, ebenso wie die Möglichkeit eines freien Meinungsaustausches, wie die Rede Günter De Bruyns zum Ausdruck brachte:

De Bruyn verliest im Kongress-Saal einen zuvor ausformulierten Text, er beginnt mit einer historischen Schilderung, der "Kriegserklärung gegen den Krieg" von Jean Paul. Aber De Bruyn wendet sich rasch der Gegenwart zu, und er findet klare Worte: "So erfreulich die Unterstützung der westeuropäischen Friedensbewegung durch die DDR auch ist, so fraglich wird ihr Nutzen bleiben, solange der Eindruck entstehen muss, dass das drüben Bejubelte hüben unerwünscht ist. Die auf Frieden gerichtete Politik der DDR, die das Bündnis mit den Friedensbewegten in aller Welt anstrebt, schädigt sich selbst, lehnt sie das Bündnisangebot unabhängiger Friedensbestrebungen im eigenen Land $a b$, das junger Christen z.B., die einen sozialen Friedensdienst fordern. Drängt man die in den Untergrund, verliert man nicht nur Friedenskräfte, sondern schädigt auch die eigene Glaubwürdigkeit [...]". (Berg 2014, 10-11)

De Bruyns damalige Reizworte lösten unterschiedliche Reaktionen aus. Die westliche Presse berichtete von Kritik ostdeutscher Bürger an ihrem Staat, während der Staatssicherheitsdienst das pazifistische Gedankengut des Treffens hervorhob und bestätigte. Denn am Beispiel von Friedensfragen wie mangelnder Abrüstung und sozialem Friedensdienst betonte der Schriftsteller Souveränitätsansprüche der DDR, indem er ihr die Verantwortung für das Ent- und Bestehen des kalten Kriegs 
auflud. (vgl. ebd.; Mählert 1998) De Bruyns Rede im Interhotel Stadt Berlin am Alexanderplatz 1981 emotionalisierte die Debatte sowohl im Kongress-Saal des Hotels als auch draußen in der Öffentlichkeit, weil sie an Widerstand und Umbruch appellierte.

Das Interhotel erweist sich hier also als Ort der politischen Opposition mit der blanken Waffe der Literatur und erinnert an jenes Widerstandsnest in Paris, an das Hotel Lutetia, wo 1937 "kommunistische, sozialdemokratische und bürgerliche Parteifunktionäre gemeinsam mit unabhängigen Intellektuellen an einem Tisch [saßen] und über die Zeit nach Hitler [sprachen]". (Jasper 1994: 10)

Das zwar isolierte jedoch musterhafte Beispiel der Berliner Friedensbegegnung von 1981 zeigt, dass nicht jeder Parallelismus zwischen Interhotels und Palästen der spätbürgerlichen Zeit unbedingt mit luxuriöser Dekadenz assoziiert werden kann. Trotzdem besteht der Reiz solcher Etablissements ebenso im Osten wie im Westen in jener Pracht und Größe, die imaginativ auf das Volk Einfluss üben. Einige Interhotels sind heute sogar Objekt des "ostalgischen" Gefühls geworden, wie im Fall des 1997 abgerissenen Interhotels Gera. 1967 anlässlich des Tags der Republik eröffnet, an dem der altgediente Kommunist und Mitglied des Politbüros des ZK der SED Albert Norden den Neubau mit der üblichen pazifistischen Rhetorik feierte (» “[...] zum Kriegsgetrommel aus Bonn, der drohenden Rückkehr des Faschismus in der BRD und der wichtigen Rolle der DDR als "Sachwalter des Friedens aller Deutschen" «, Kaden 2017), wurde das Interhotel zum Erinnerungsort für die damaligen Mitarbeiter und Mitarbeiterinnen, die im Oktober 2017 ein Wiedersehen fünfzig Jahre nach dessen Eröffnung zu Zwecken der "ostalgischen" Erinnerung (Photos- und 
Anekdotenaustausches) organisierten, und deren Teilnahme an den Kommentaren auf dem der Initiative gewidmeten Blog nachzulesen sind (vgl. https://interhotelgera.wordpress.com/ Letzter Abfruf: 21.11.2019). Auch für Gäste soll es in Erinnerung geblieben sein, wie folgende Postkarte von 1972 dokumentiert:

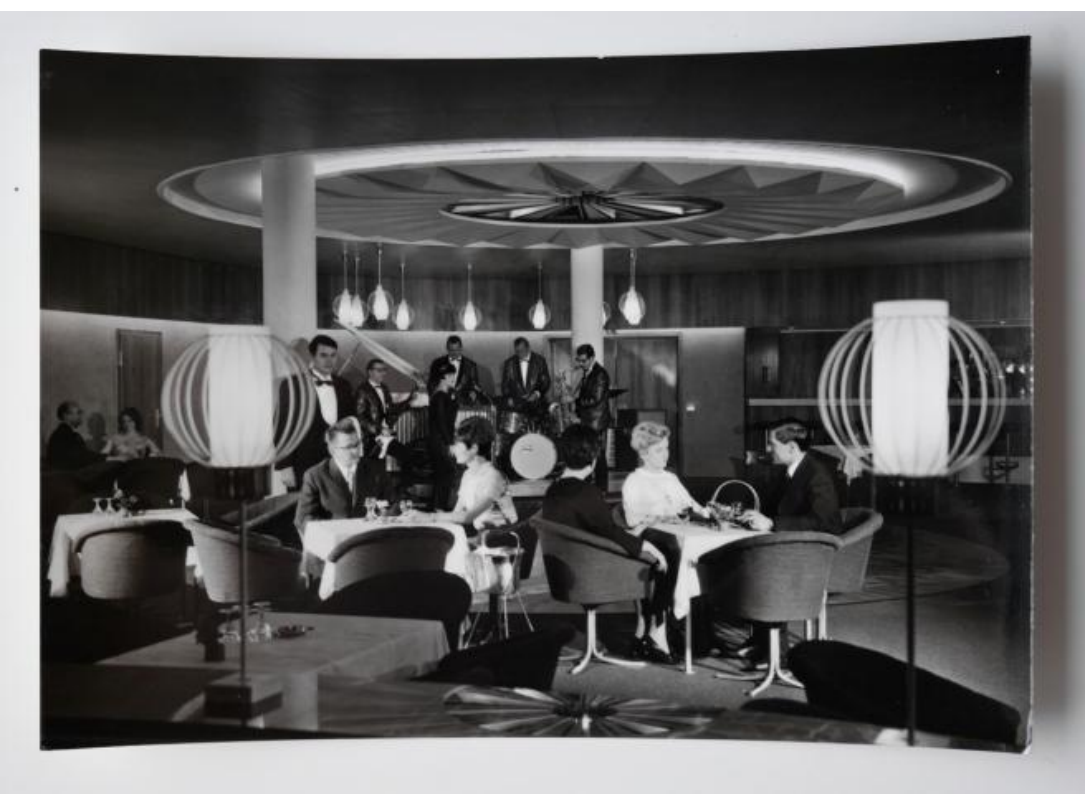

Die Tanzbar Interhotel "Gera", vermutlich Richtung 1972, in einem Jahr also, in dem es der Uve-SchikoraCombo gelang, Prog-Rock mit Schlager zu kombinieren (u.a. im Duett mit Frank Schöbel) und populär zu machen. $\mathrm{Ob}$ sie auch einmal auf dieser Bühne auftraten, ist nicht überliefert. (Kaden 2017, letzter Zugriff: 22.11.2019) 
Die Karte, die von einer Exkursion der Schulfunktionäre des Kreises Bitterfeld zeugt und im Hintergrund ein Barquartett aus der ČSSR zeigt, stellt die Tanzbar des Interhotels Gera dar, die sehr beliebt war sowohl für Hotelgäste als auch für Gäste außerhalb der gastlichen Einrichtung, es sei denn, dass »die postalisch nicht gelaufene Karte« (vgl. Kaden 2017) als ein Beweis »künstlicher Harmonie« zu betrachten sei. Letzteres spräche wenigstens für den »internationalen Charakter ästhetischer Leitbilder « (ebd.), dank derer die DDR auf Augenhöhe mit dem Rest Europa stehen konnte. Ob das Innovation oder (Selbst)betrug war, es ist nun mal Geschichte geworden. Der Bezug auf die Kombination von Prog-Rock mit Schlager ist ein Hinweis auf die Popkultur, die in den Interhotels Gästen als Kompromisslösung gegönnt wurde, um Zerstreuung und Unterhaltung auch fern vom bürgerlichen Luxus anstelle engagierten (d.h. kritischen) Verhaltens anzubieten. Auf das zwielichtige Potential von Tanzbars in Interhotels machte die Presse bereits zu Beginn von deren Existenz aufmerksam, indem sie es mit den Krimi-TV-Serien der 1950er Jahre verknüpfte (vgl. Kader 2017).

Aus diesem Blickwinkel sind Interhotels wiederum Bindeglied zur deutschen Kultur der Vergangenheit. Siegfried Kracauer hatte bereits 1925 die Hotel-Halle mit dem Detektivroman und mit dem Vakuum der Säkularisierung in Verbindung gebracht (Kracauer 1979). Dass aber das letzte Interhotel der DDR, das Grand Hotel in der Friedrichstraße, 1987 als repräsentatives Spitzenhotel errichtet zugleich aber auch als Bühne und Drehort von Krimi-TV-Serien und DEFA-Filmen war, wird von einer MDR-Dokumentation vom 18.12.2018 bestätigt: 


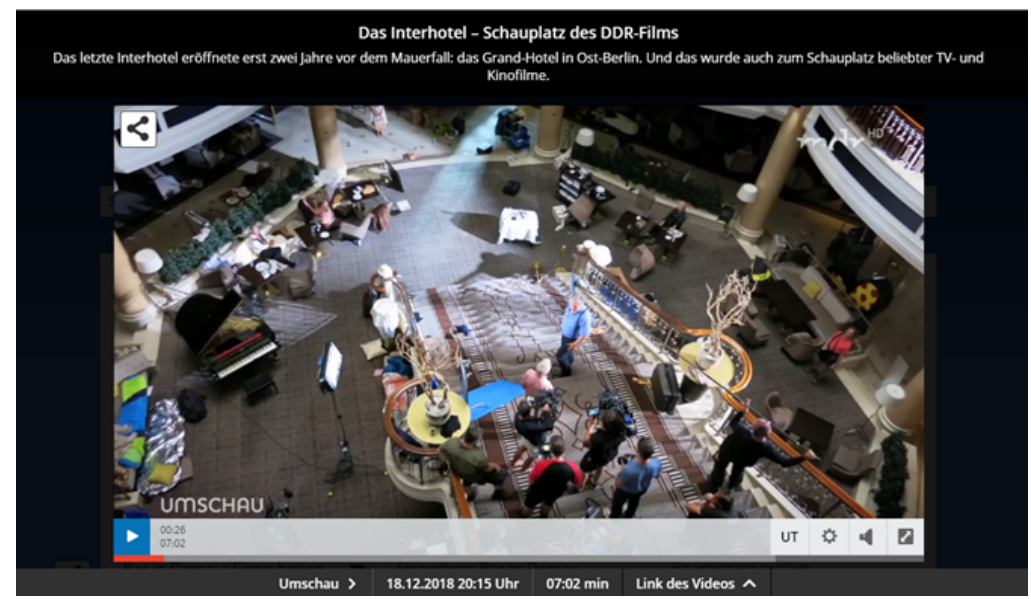

Das Haus, das seit der Wiedervereinigung zur Gruppe Westin gehört und in "The Westin Grand Berlin" umbenannt wurde, sollte schon zur Eröffnung für seine Großzügigkeit beeindrucken, vor allem wegen des großen Treppenhauses. Die Dokumentation verrät auch die Namen einiger Persönlichkeiten, die dort zu Gast waren, u.a. Rainer Calmund, Bud Spencer, Bruce Springsteen, Loriot. Der Wende-Krimi "Unter Brüdern" (Erstaustr. 28.10.1990) wurde dort, als Koproduktion mit ARD Tatort 110 gedreht und vereinte den Ostdeutschen Schauspieler Andreas Schmidt-Schaller mit seinem westdeutschen Kollegen Götz George: Eine deutsch-deutsche KrimiVereinigung, die programmatisch ein Interhotel als Handlungsort hatte.

Die hier vorgestellte Übersicht zu den Interhotels und deren Bezüge zur Literatur macht schließlich die Frage relevant, ob ihre Gründung zur Überwindung der Hallstein-Doktrin beitrug, indem sie - laut Torben Gülstorff - »nationale ökonomische und internationale 
geostrategische Interessen verfolgten «? (Gülstorff 2017) Ob sie nur einen "deutschen Sonderweg" im Bereich der Außenpolitik darstellten? Insofern gelten Interhotels heute - 30 Jahre nach der Wiedervereinigung - als Beweis dafür, dass die Voraussetzungen des DDR-Beitritts zur BRD bereits durch ihre Entstehung in den 1960er Jahren geschaffen wurden. Interhotels sind als eine bürgerliche Falle entstanden, in die schließlich der Staat selbst gegangen ist. 


\section{Literatur}

Benveniste, Emile. Il vocabolario delle istituzioni indoeuropee, Torino, Einaudi, 1976.

Berg, Stefan, Günter De Bruyn. Landgang: Ein Briefwechsel. Frankfurt a.M.: Fischer, 2014.

"Das Interhotel - Schauplatz des DDR-Films". MDRDokumentation, abrufbar unter https: / / www.mdr.de/suche/video-259882_zc-

13f2fa39_zs-8454ba72.html (Letzter Zugriff: 30.11.2019. Das Video ist bis zum 18.12.2019 unter der angegebenen Adresse verfügbar).

Christ, Peter. "Valuta-Republik". Die Zeit 28 (1986), https: / / www.zeit.de/1986/28/ die-valuta-republik (Letzter Zugriff: 8.11.2019)

Delius, Friedrich C. und Peter J. Lapp. Transit Westberlin. Berlin: Ch. Links, 1999.

Dunte, Andreas. "Ökonomie siegt über Prinzipien" Matthias Judt im Interview. Leipziger Volkszeitung 1.3.2014, S. 3.

Fröhlich, Jörg. Ein Hotel im Lustgarten in Potsdam. das Interhotel Potsdam in historischer Umgebung von 1967 bis heute. Norderstedt: Books on Demand GmbH, 2012.

Foucault, Michel. "Von anderen Raumen". In Raumtheorie. Grundlagentexte aus Philosophie und Kulturwissenschaften. Hrsg. v. J. Dünne u. S. Günzel. Frankfurt a.M.: Suhrkamp, 2006, S. 317-329.

Große, Herbert E.. Poppichs Flucht (German Edition), Kindle, ${ }^{2} 2015$.

Gülstorff, Torben. "Die Hallstein-Doktrin - Abschied von einem Mythos". In: Deutschland Archiv, 9.8.2017, www.bpb.de/253953. (Letzter Zugriff: 26.11.2019). 
"Interhotel". In DDR-Lexikon. http://www.ddrwissen.de/wiki/ddr.pl?Interhotel (Letzter Zugriff: 2.12.2019)

Interhotel

Gera.

Blog: https: / / interhotelgera.wordpress.com/ (Letzter Abfruf: 21.11.2019).

Heinrich, Marie-Thèrése. “ 'Operativ interessante Gäste'. Der Tourismus als Sicherheitsproblem in den Nordbezirken der DDR 1972-1989". In Dem Gedächtnis eine Erinnerung: Der Mauerfall von 1989 und seine Relevanz für kommende Generationen. Hrsg. v. Heiner Timmermann. Berlin, LIT, 2016, S. 63-78.

Kaden, Ben. "Die Tanzbar, die es gab". Retraceblog. Materialsammlung: Bibliografie $\mathrm{zu}$ Kunst und Architektur in der DDR mit dem Schwerpunkt baubezogene Kunst und Kunst im öffentlichen Raum: https: / / retraceblog.wordpress.com/2017/02/12/tanzb ar/ (Letzter Zugriff: 22.11.2019)

Körner, Katarina. Leitungsstile in der DDR. Ein Vergleich der Eliten in Partei, Industrie und Dienstleistungszweig 1971 bis 1989. Berlin: Metropol, 2016.

Kracauer, Siegfried. "Die Hotelhalle" (1925). In Der Detektiv-Roman. Ein philosophischer Traktat. (Schriften, Bd. I). Frankfurt am Main: Suhrkamp, 1971, S. 128-137.

Lukas, Julius. "Sieg der Sterne". Die Zeit 33 (2016) vom 4.8.2016.

Mählert, Ulrich. Kleine Geschichte der DDR. München: Beck, 1998.

Matthias, Bettina. The Hotel as Setting in Early Twentiethcentury German and Austrian Literature: Checking in to Tell a Story, New York: Camden House, 2006.

Pohlmann, Friederike. Hotel der Spione. Das "Neptun" in Warnemunde. Schwerin: Landesbeauftragte für 
Mecklenburg-Vorpommern für die Unterlagen der Staatssischerheitsdienst der ehemaligen DDR, 2017.

Pragal, Peter. "Vertraute Fremde. Wie ein West-Journalist Ost-Berlin erlebte". In Ost-Berlin: 30 Erkundungen. Hrsg. von Jürgen Danyel. Berlin, Ch. Links, 2019, S. 383-396.

Seipp, Bettina. "Was aus den Hotels des Sozialismus wurde". Die Welt, 26.10.2009, abrufbar unter http: / / www.welt.de/ 4984328

[o. Verf.]. “Wer wen melkt". Der Spiegel 38 (1990), S. 131133.

[o. Verf.]. “Ich sollte Herren im Hotel Aushorchen”. Der Spiegel 47 (1976), S. 67-81.

Seger, Cordula. Grand Hotel, Schauplatz der Literatur. Köln, Böhlau 2005.

Seger, Cordula und Reinhard G. Wittmann (Hg.). Grand Hotel. Bühne der Literatur. München: Dolling \& Galitz, 2007.

Taubert, Klaus. DDR-Spottgedichte. Blog: http://www.klaus-taubert.de abrufbar unter https: / / klaustaubert.wordpress.com/2014/01/22 / ddr-spottgedichte / (Letzter Zugriff: 2.12.2019)

Wolf, Christa. Kindheitsmuster. Hrsg. v. Sonja Hilzinger. München: Luchterhand 2000.

Wolter, Heike. "Ich harre aus im Land und geh, ihm fremd". Die Geschichte des Tourismus in der DDR. Frankfurt a.M.: Campus, 2009.

Ulrich, Silvia. "De muta eloquentia. La signorina Else di Arthur Schnitzler tra monologo interiore, pantomima e film muto". In Il silenzio. Pause eloquenti della parola. Hrsg. v. Valeria Gianolio. Turin: Tirrenia Stampatori/Neos Edizioni, 2010, S. 121-133.

Ulrich, Silvia. "Hotels in der Literatur als Nicht-Orte: Körperliche Verortungen an drei Beispielen aus dem 20. Jahrhundert". In Provisorische und Transiträume: 
Raumerfahrung Nicht-Ort. Hrsg. v. Miriam Kanne. Berlin: LIT, 2013, S. 91-106.

Ulrich, Silvia. "Asylsuchende Weltbürger, heimatlose Hotelpatrioten und Verwandtes. Zur Wohnsymbolik bei Stefan Zweig, Joseph Roth und Franz Kafka". In "Das rechte Gespräch: keine( $r$ ) ringt nach Geltung, aber jede( $r$ ) kommt zur Geltung!". Hrsg. v. Mariana Virginia Lăzărescu u. Delia Cotârlea. Berlin: Wissenschaftlicher Verlag, 2018, S. 94-110. 